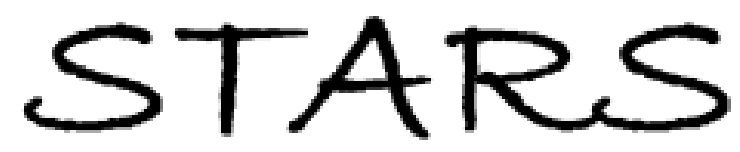

University of Central Florida

STARS

$1-1-1992$

\title{
Simultaneous Measurement Of Electron And Hole Escape Times From Biased Single Quantum-Wells
}

\author{
J. A. Cavaillès \\ D. A. B. Miller \\ J. E. Cunningham \\ P. Li Kam Wa \\ University of Central Florida \\ A. Miller \\ University of Central Florida
}

Find similar works at: https://stars.library.ucf.edu/facultybib1990

University of Central Florida Libraries http://library.ucf.edu

This Article is brought to you for free and open access by the Faculty Bibliography at STARS. It has been accepted for inclusion in Faculty Bibliography 1990s by an authorized administrator of STARS. For more information, please contactSTARS@ucf.edu.

\section{Recommended Citation}

Cavaillès, J. A.; Miller, D. A. B.; Cunningham, J. E.; Wa, P. Li Kam; and Miller, A., "Simultaneous Measurement Of Electron And Hole Escape Times From Biased Single Quantum-Wells" (1992). Faculty Bibliography 1990s. 413.

https://stars.library.ucf.edu/facultybib1990/413

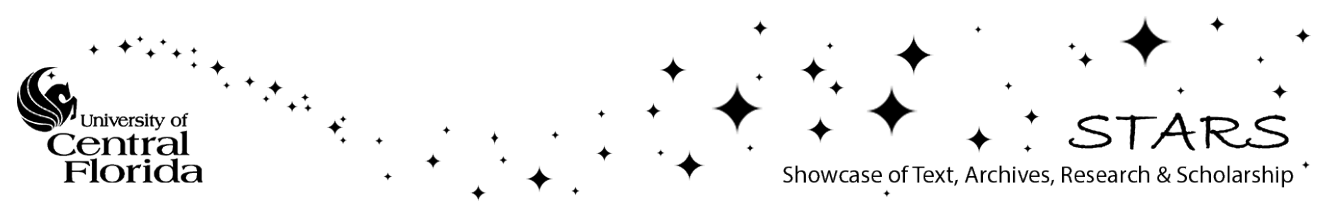




\section{Simultaneous measurement of electron and hole escape times from biased single quantum wells}

Cite as: Appl. Phys. Lett. 61, 426 (1992); https://doi.org/10.1063/1.107903

Submitted: 10 February 1992 . Accepted: 27 May 1992 . Published Online: 04 June 1998

J. A. Cavaillès, D. A. B. Miller, J. E. Cunningham, P. Li Kam Wa, and A. Miller
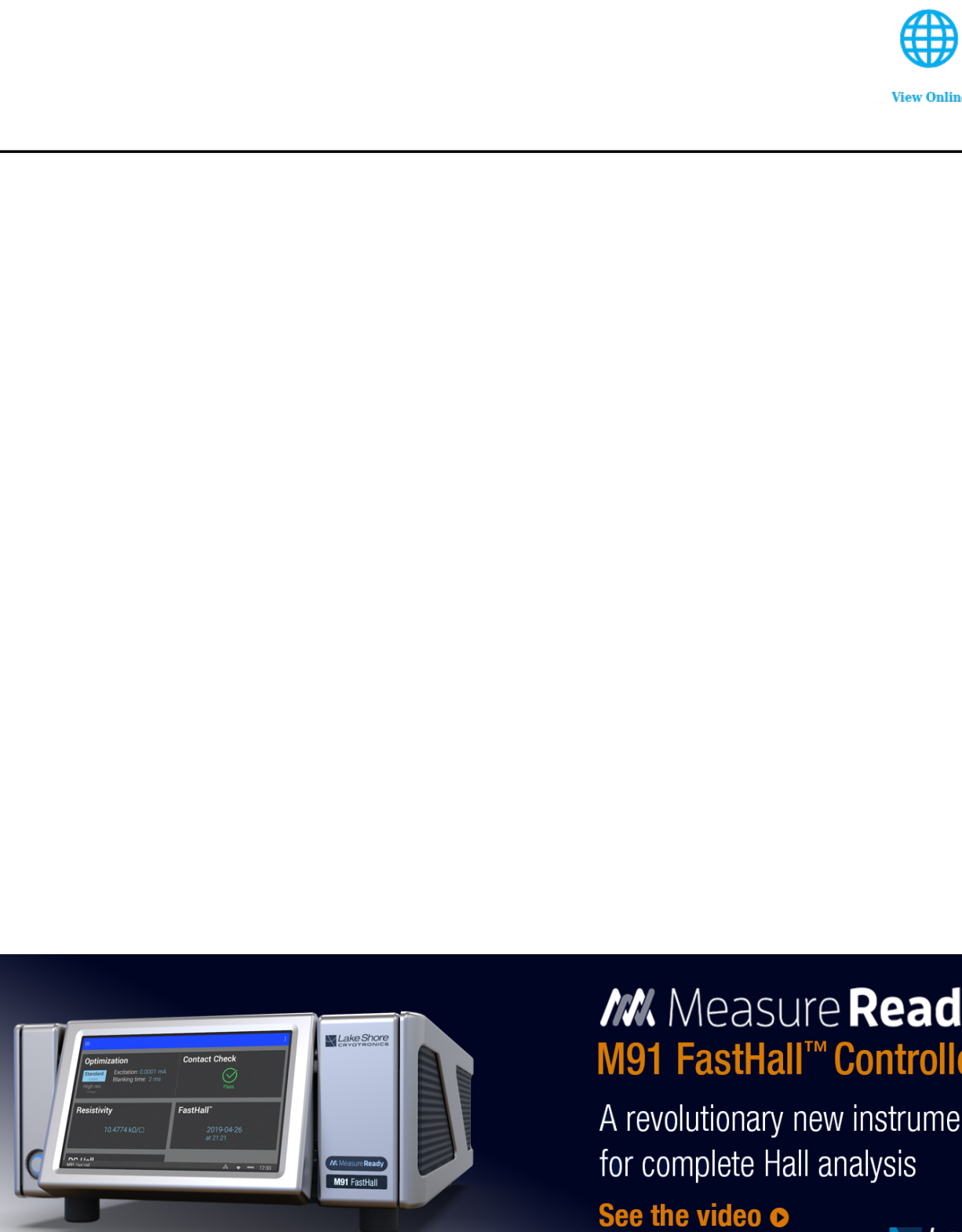

\section{Measure Ready} M91 FastHall ${ }^{\text {TM }}$ Controller

A revolutionary new instrument for complete Hall analysis See the video 0 


\title{
Simultaneous measurement of electron and hole escape times from biased single quantum wells
}

\author{
J. A. Cavailles, a) D. A. B. Miller, and J. E. Cunningham \\ AT\&T Bell Laboratories, Crawfords Corner Rd, Holmdel, New Jersey 07733 \\ P. Li Kam Wa and A. Miller \\ Center for Research in Electro-Optics and Lasers, University of Central Florida, \\ 12424 Research Parkway, Orlando, Florida 32826
}

(Received 10 February 1992; accepted for publication 27 May 1992)

\begin{abstract}
Picosecond pump probe measurements on waveguides containing a single GaAs/GaAlAs quantum well have enabled us to determine for the first time both electron and hole escape times from biased quantum wells. Contributions from excitonic saturation and field screening by photogenerated carriers can be clearly identified in the nonlinear transition signal and are quantitatively modeled. Preliminary analysis suggests that thermally assisted escape is dominant at room tempcrature, but discrepancies are found with conventional thermionic emission models.
\end{abstract}

Carrier escape times from quantum wells are key parameters for the switching speed of self-electro-optic effect devices (SEEDs). ${ }^{1-3}$ These devices are based on $p-i-n$ diodes that contain quantum wells in their intrinsic region and combine the detecting and light modulating properties of such diodes. Carrier escape dynamics influence both of these aspects of SEED device operation. Detection response times are ultimately limited by the speed at which photogenerated carriers leave the wells. Moreover, the optical intensity modulation contrast is degraded when the carrier concentration inside the well is high, due to excitonic absorption saturation ${ }^{1}$ and field screening. ${ }^{4,5}$ Fast escape times could also prevent carrier accumulation in the wells under strong illumination. These arguments have motivated many investigations on the mechanism ${ }^{6}$ of carrier escape from quantum wells. Most such studies have concentrated on electron escape; little is known about the behavior of the holes. To our knowledge, the only information concerning hole sweep out from quantum wells has been obtained with a temporal resolution limited to $\sim 100$ ps. 5,7

In this letter, we present simultaneous measurements of both hole and electron escape times from electrically biased quantum wells. We performed optical pump and probe experiments on waveguides containing a single quantum well, with a resolution $\sim 1$ ps.

There are primarily two mechanisms by which the photogenerated carriers can influence the absorption coefficient of a biased quantum well: excitonic saturation and screening of the applied electric field by photogenerated carriers. Excitonic saturation is due to various many-body mechanisms, ${ }^{8}$ dominated by the process of band or phase space filling, ${ }^{9}$ by which photogenerated carriers occupy available states and inhibit band-to-band or excitonic optical transitions. Field screening is an electroabsorption process: photogenerated carriers screen the applied electric field, which, through the quantum confined Stark effect ${ }^{9}$

\footnotetext{
a) Permanent address: Alcatel Alsthom Recherche, Route de Nozay, 91450 Marcoussis, France.
}

(QCSE), shifts the excitonic resonances and therefore modifies the absorption of the quantum well. An cssential feature of our experimental results is that both effects can be clearly distinguished, which enables us to extract both electron and hole escape times.

Results were obtained on a pair of complementary samples enabling a clearer attribution of their respective contributions to the nonlinear signal. The schematic structure of the samples is shown in Fig. 1. These are single mode $\mathrm{Ga}_{0.7} \mathrm{Al}_{0.3} \mathrm{As} / \mathrm{Ga}_{0.75} \mathrm{Al}_{0.25} \mathrm{As}$ double heterostructure $p-i-n$ waveguides. The $\mathrm{Ga}_{0.75} \mathrm{Al}_{0.25} \mathrm{As}$ waveguide core is 0.5 $\mu \mathrm{m}$ thick; the bottom $\mathrm{Ga}_{0.7} \mathrm{Al}_{0.3} \mathrm{As}$ confining layer thickness is $1 \mu \mathrm{m}$ and that of the upper one is $1.2 \mu \mathrm{m}$. The waveguide core is located in the middle of the $1 \mu \mathrm{m}$ thick intrinsic region. The structures were grown by molecular beam epitaxy on $n^{+}$GaAs substrates and were capped by a thin $\left(100 \AA\right.$ ) $p^{++}$GaAs layer (not shown in Fig. 1) for good ohmic contacts.

The quantum well is in the intrinsic region, slightly outside the waveguide core $(0.17 \mu \mathrm{m}$ below $)$. By decreasing the optical mode quantum well overlap $(0.005)$, this position gives low modal absorption that allows conveniently long $(\sim 300-500 \mu \mathrm{m})$ samples. In each case, the quantum well consists of a $95 \AA$ thick GaAs layer sandwiched between $200 \AA$ thick GaAlAs barriers with a $40 \%$ $\mathrm{Al}$ fraction on one side and $20 \%$ on the other. For both samples, zero-field heavy hole and light hole exciton wavelengths were 849 and $845 \mathrm{~nm}$, respectively.

With respect to the quantum well, the high $\mathrm{Ga}_{0.6} \mathrm{Al}_{0.4} \mathrm{As}$ barrier is toward the $p$-layer in sample $\mathrm{A}$ and toward the $n$-layer in sample $\mathbf{B}$. Under reverse bias, this leads to the band diagrams shown in Fig. 1. In sample A, it will be relatively easy for the electrons to escape and hard for the holes. The situation is reversed in sample $\mathbf{B}$.

Lateral light confinement was obtained by chemically etching $4 \mu \mathrm{m}$ wide ribs $(0.85 \mu \mathrm{m}$ depth $)$ on the top surface. Ribs were then covered by $30 \mu \mathrm{m}$ wide, $0.2 \mu \mathrm{m}$ thick $\mathrm{Au} / \mathrm{Zn} / \mathrm{Au} p$-ohmic contact electrodes, and a $\mathrm{AuGe} / \mathrm{Ni} /$ $\mathrm{Au} n$-ohmic contact was deposited on the back surface of the sample. 

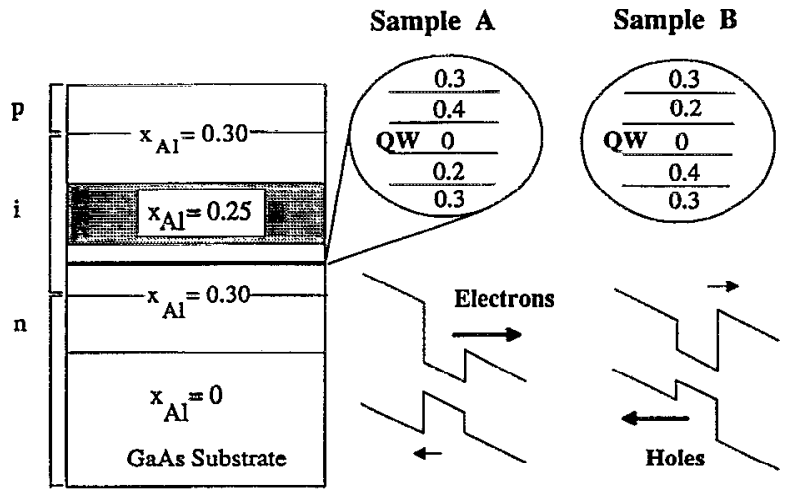

FIG. 1. Schematic structure of the samples. Enlarged sections show the quantum well region for samples $\mathrm{A}$ and $\mathrm{B}$, as well as the $\mathrm{Al}$ fraction of the different layers around it. Quantum wells are $95 \AA$ thick and the $\mathrm{Ga}_{0.6,0.8} \mathrm{Al}_{0.4,0.2} \mathrm{As}$ barriers are $200 \AA$ thick. The corresponding band diagrams when the $p-i-n$ diode is reverse biased are shown for both samples; arrows symbolize carrier escape, the longer the arrow, the faster the escape.

We used a synchronously pumped dye (Styryl 9) laser, tunable in the $810-880 \mathrm{~nm}$ range and delivering $0.7-1 \mathrm{ps}$ long pulses with a $80 \mathrm{MHz}$ repetition rate, in a conventional pump-probe setup (see Ref. 6). Light was coupled into and collected from the waveguide by means of microscope objectives.

The pump and probe beams were orthogonally polarized, so that light from the pump could be easily suppressed at the output with a polarizer. In all experiments described here, the pump was TE polarized (parallel to epilayers) and the probe TM. The estimated average optical powers coupled into the waveguide were $\sim 30 \mu \mathrm{W}$ for the pump and $3 \mu \mathrm{W}$ for the probe, giving a maximum photocarrier density of a few times $10^{10} \mathrm{~cm}^{-2}$.

We measured the changes in the transmission of the probe as a function of its delay relative to the pump at several wavelengths and for various biases (between +3 and $-10 \mathrm{~V}$ ). Since TM polarized light only probes optical transitions involving light holes, ${ }^{9}$ we have restricted ourselves to wavelengths around the light hole exciton. Figure 2 shows the results obtained for both samples, for a $6 \mathrm{~V}$ reverse bias at different wavelengths. For this bias the light hole exciton wavelength is around $855 \mathrm{~nm}$. A positive signal corresponds to an increase in transmission for the probe.

For sample $\mathrm{A}$, the sign of the signal changes with wavelength. It is positive at $859 \mathrm{~nm}$ and negative at 845 nm. Apart from the sign, the signal at both these wavelengths shows very similar features; it rises in about 5 ps and decays with a time constant on the order of $60 \mathrm{ps.}$

An increase in the electric field across a quantum well induces an increase in absorption at wavelengths above the exciton resonance and a decrease below (the wavelength at which the electroabsorption changes sign is actually slightly longer than the exciton resonance wavelength). This strongly suggests that the transmission changes observed for sample $\mathrm{A}$ are mainly due to electroabsorption induced by field screening. We found that the pump-probe signal changes sign precisely at the same wavelength as the

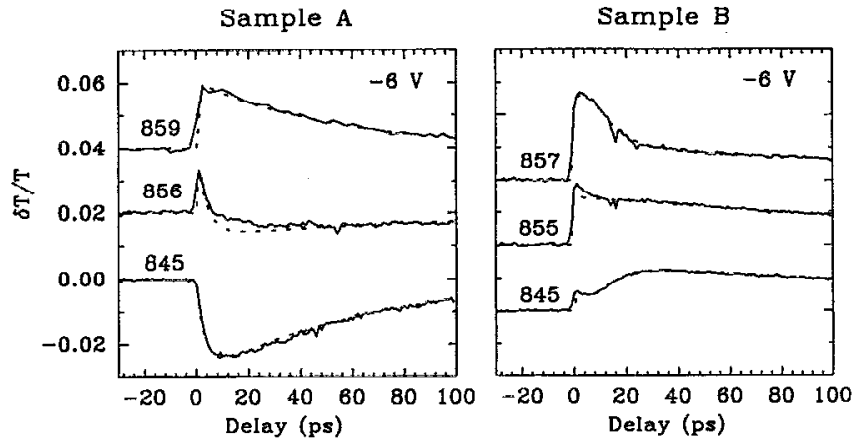

FIG. 2. Relative changes in the probe transmission induced by the pump as a function of the pump-probe delay, for both samples $\mathrm{A}$ and $\mathrm{B}$. The reverse bias across the diodes was $6 \mathrm{~V}$. Traces are recorded at the wavelength indicated (nm) in the figure. Solid curves are experimental. Dotted curves are theoretical fits. Traces are offset for clarity.

differential electroabsorption. At $-6 \mathrm{~V}$, the differential electroabsorption is zero at about $856 \mathrm{~nm}$. The fast signal detected at this wavelength (Fig. 2) is due to excitonic saturation. It rises instantaneously (within experimental resolution) and decays in about 5 ps.

For sample $B$ the zero-electroabsorption wavelength (for a $6 \mathrm{~V}$ reverse bias) is around $855 \mathrm{~nm}$. The pump probe signal at this wavelength (Fig. 2) is due to excitonic saturation. It rises instantaneously and decays exponentially with a time constant around 200 ps. At other wavelengths, the field screening contribution shows itself most clearly in the first 30 ps or so. As one may expect, this supplementary component is positive at $857 \mathrm{~nm}$ and negative at $845 \mathrm{~nm}$. At $845 \mathrm{~nm}$, it is very clear that the electroabsorption component (negative at this wavelength) reaches a maximum (minimum in the signal) between 5 and 10 ps.

The same generic wavelength dependence of the pumpprobe signal was observed for both samples for reverse biases greater than $1 \mathrm{~V}$. At each bias, the wavelength was adjusted so that the field screening contribution was negative for sample A and positive for sample B. From these results, we determined the carrier escape times as described in the following.

Electron escape times are directly deduced from the excitonic saturation decay (carrier recombination is known to be negligible ${ }^{6}$ ), because this process is here essentially due to electrons present in the wells, and much less to the holes. We already mentioned that, since the probe is TM polarized, its absorption only involves lighthole transitions, and heavy holes cannot contribute to band filling for this polarization. Most of the holes will end up as heavy holes on timescales of interest here, both because of the lower energy of the heavy hole band and its possibly larger density of states. Hence, we expect little band or phase space filling from holes here.

By contrast, the field screening component is sensitive to both electrons and holes. This process is much more difficult to analyze than saturation, because its dynamics involves transport of photogenerated electrons and holes. We based our analysis on a simple model which we de- 




FIG. 3. Electron (circles) and hole (triangles) escape times for sample A (filled symbols) and B (open symbols), as a function of the applied electric field.

scribe only briefly here (details will be presented elsewhere).

We assumc that transmission changes are linearly proportional to changes in electric field at the quantum well. The change in electric field created by the photogenerated charges at a given time is computed by solving the 1dimensional Poisson equation, including both the charges remaining in the quantum well and the space charge from escaped carriers drifting across the intrinsic region (we neglect diffusion and assume the same saturated drift velocity for the electrons and holes). In this simple model, diffusive conduction (i.e., the process by which carriers in the electrodes redistribute themselves to wash out any electrical potential nonuniformity, see Ref. 10), is almost instantaneous, due to the highly conductive metallic electrodes and the small lateral dimensions $(\sim 4 \mu \mathrm{m})$ of the waveguides. This actually locks the potential drop across the intrinsic region to its equilibrium value, even on a picosecond timescale. With this constraint, we can compute the electric field changes across the quantum well as a function of time.

This model fits very well with the experimental results (dotted curves in Fig. 2). Adjustable parameters were the electron and hole escape times, and the amplitudes of saturation and field screening contributions. A carrier saturated drift velocity of $0.8-1 \times 10^{7} \mathrm{~cm} / \mathrm{s}$ was assumed. We estimate a $10 \%-20 \%$ uncertainty in the escape times deduced from the fits.

Figure 3 shows the electron and hole escape times as a function of the applied electric field for both samples. The electronic escape times are in qualitative agreement with previously measured values on MQWs. ${ }^{6}$ Escape times for both carriers decay approximately exponentially with electric field. The slopes, in a semi-log plot (Fig. 4), of the escape time versus electric field curves are strikingly similar for both samples and both types of carriers. This suggests that, among the two main escape mechanisms, ${ }^{6}$ thermionic emission and field-induced quantum tunneling, the first one is dominant. Field-induced tunneling would give a nonexponential dependence on field ${ }^{6}$ and a different behavior for electrons and holes. On the other hand, conventional thermionic emission theory ${ }^{7}$ gives, for the emission time

$$
\tau_{\mathrm{th}}=\left(\frac{2 \pi m W^{2}}{k T}\right)^{1 / 2} \exp \left(\frac{E_{b}-E-e F W / 2}{k T}\right)
$$

where $W$ is the well width, $E_{b}$ the barrier height, $F$ the electric field, $m$ the particle's mass, and $E$ its energy. This equation reproduces some features of the experimental values. The escape time decays exponentially with field, with a rate independent of the effective mass. The experimental value of this rate is $35 \pm 510^{-3} \mathrm{~cm} / \mathrm{kV}$, which is within a factor of two of the theoretical value $e W / 2 k T\left(=19 \times 10^{-3}\right.$ $\mathrm{cm} / \mathrm{kV}$ at room temperature). However, (1) fails to reproduce the absolute values of the sweep-out times. For a $\mathrm{Ga}_{0.8} \mathrm{Al}_{0.2} \mathrm{As}$ barrier and for a field of $1.5 \times 10^{4} \mathrm{~V} / \mathrm{cm}$, theory ${ }^{6}$ predicts 14 and 3.5 ps escape times for the electrons and holes, respectively, whereas we measure about $20 \mathrm{ps}$ for the electrons (sample A), and $50 \mathrm{ps}$ for the holes (sample B). The discrepancy observed for hole escape times is not yet understood.

More experimental work is needed to assess carrier escape mechanisms more precisely. We believe that the single quantum well method and analysis presented here, which allowed the first determination of escape times for both types of carriers, provides a useful conceptual framework for future studies.

We gratefully acknowledge help from G. E. Doran and C. A. Burrus, as well as many stimulating discussions with J. Feldmann, T. C. Damen, and G. D. Boyd. P. Li Kam Wa and A. Miller acknowledge support from DARPA.

${ }^{1}$ D. A. B. Miller, Opt. Quantum Electron. 22, 61 (1990).

${ }^{2}$ J. Feldmann, K. Goossen, D. A. B. Miller, A. M. Fox, J. E. Cunningham, and W. Y. Jan, Appl. Phys. Lett. 59, 66 (1991).

${ }^{3}$ A. M. Fox, D. A. B. Miller, G. Livescu, J. E. Cunningham, J. E. Henry, and W. Y. Jan, Appl. Phys. Lett. 57, 2315 (1991).

${ }^{4}$ I. Bar-Joseph, G. Sucha, D. A. B. Miller, D. S. Chemla, B. I. Miller, and U. Koren, A.ppl. Phys. Lett. 52, 51 (1988).

${ }^{5}$ T. H. Wood, J. Z. Pastalan, C. A. Burrus, Jr., B. C. Johnson, B. I. Miller, J. L. de Miguel, U. Koren, and M. G. Young, Appl. Phys. Lett. 57, 1081 (1990).

${ }^{6}$ For a recent review see; A. M. Fox, D. A. B. Miller, G. Livescu, J. E. Cunningham, and W. Y. Jan. IEEE J. Quantum Electron. 27, 2281 (1991).

${ }^{7}$ H. Schneider and K. v. Klitzing, Phys. Rev. B 38, 6160 (1988).

${ }^{8}$ D. S. Chemla and D. A. B. Miller, IEEE J. Quantum Electron. 2, 1155 (1985).

${ }^{9}$ S. Schmitt-Rink, D. S. Chemla, and D. A. B. Miller, Adv. Phys. 38, 89 (1989).

${ }^{10}$ G. Livescu, A. M. Fox, D. A. B. Miller, T. Sizer, W. H. Knox, J. E. Cunningham, A. C. Gossard, and J. H. English, Semicond. Sci. Technol. 5, 549 (1990). 